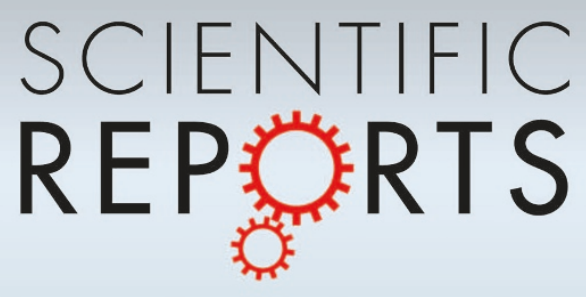

OPEN

SUBJECT AREAS:

TWO-DIMENSIONAL

MATERIALS

DESIGN, SYNTHESIS AND PROCESSING

SURFACES, INTERFACES AND

THIN FILMS

GRAPHENE

Received

7 August 2013

Accepted

2 September 2013

Published

18 September 2013

Correspondence and requests for materials should be addressed to S.J. (jslhusł@hotmail.

com)

\section{Room temperature rubbing for few-layer two-dimensional thin flakes directly on flexible polymer substrates}

\author{
Yan Yu, Shenglin Jiang, Wenli Zhou, Xiangshui Miao, Yike Zeng, Guangzu Zhang \& Sisi Liu
}

School of Optical and Electronic Information, Huazhong University of Science and Technology, Wuhan, 430074, PR China.

The functional layers of few-layer two-dimensional (2-D) thin flakes on flexible polymers for stretchable applications have attracted much interest. However, most fabrication methods are "indirect" processes that require transfer steps. Moreover, previously reported "transfer-free" methods are only suitable for graphene and not for other few-layer 2-D thin flakes. Here, a friction based room temperature rubbing method is proposed for fabricating different types of few-layer 2-D thin flakes (graphene, hexagonal boron nitride (h-BN), molybdenum disulphide $\left(\mathrm{MoS}_{2}\right)$, and tungsten disulphide $\left.\left(\mathrm{WS}_{2}\right)\right)$ on flexible polymer substrates. Commercial 2-D raw materials (graphite, $\mathrm{h}-\mathrm{BN}, \mathrm{MoS}_{2}$, and $\mathrm{WS}_{2}$ ) that contain thousands of atom layers were used. After several minutes, different types of few-layer 2-D thin flakes were fabricated directly on the flexible polymer substrates by rubbing procedures at room temperature and without any transfer step. These few-layer 2-D thin flakes strongly adhere to the flexible polymer substrates. This strong adhesion is beneficial for future applications.

ecent developments in electronics, photonics, and mechanics have increased the demand for mono- and few-layer two-dimensional (2-D) thin flakes ${ }^{1,2}$, such as graphene ${ }^{1-4}$, hexagonal boron nitride $(\mathrm{h}-\mathrm{BN})^{5-8}$, molybdenum disulphide $\left(\mathrm{MoS}_{2}\right)^{6-12}$, and tungsten disulphide $\left(\mathrm{WS}_{2}\right)^{6-11}$. The novel properties ${ }^{13-19}$ of these materials enable their use for many applications $s^{1-12,20-23}$.

Within this broad range of applications, stretchable films that are composed of mono- and few-layer 2-D thin flake functional layers on flexible polymers have attracted interest ${ }^{22,24,25}$. Several methods, including liquid-phase exfoliation, chemical vapour deposition (CVD), and chemical approaches, have been studied for fabricating mono- and few-layer 2-D thin flakes on polymer substrates ${ }^{26-35}$.

However, these approaches are "indirect" fabrication approaches ${ }^{26}$. After mono- and few-layer 2-D thin flakes are prepared in liquids ${ }^{27-29}$ or on templates ${ }^{30-32}$, an additional step is required to "transfer" the 2-D thin flakes to the polymer substrate $e^{26,33}$. Thus, recently developed "transfer-free" processes for attaching graphene and its 2-D analogues on polymer substrates are important ${ }^{26,33-35}$. However, these "transfer-free" methods have only been applied to graphene. In addition, these methods are unsuitable for use with other 2-D thin flakes. Furthermore, most of these methods require processing temperatures that are greater than $150^{\circ} \mathrm{C}$, which common polymers cannot withstand.

Here, a room temperature rubbing method that is based on the friction and self-lubricating properties of the 2D layered materials is proposed for the transfer-free fabrication of few-layer 2-D thin flakes on polymer substrates. Different types of few-layer 2-D thin flakes were directly fabricated on flexible polymer substrates with commercial two-dimensional raw materials (composed of thousands graphite, h-BN, $\mathrm{MoS}_{2}$, and $\mathrm{WS}_{2}$ atomic layers) after performing rubbing procedures for several minutes at room temperature with no transfer step.

\section{Results}

Advantages of rubbing method. The experiment details are given in Figure 1 and the Methods section.

This rubbing method has the following important advantages: (1) producing a variety of few-layer 2-D thin flakes, (2) the rapid fabrication procedures (minutes) at room temperature, (3) the direct fabrication on flexible polymer substrates without any transfer step, (4) low raw material costs, and (5) a strong surface adhesion between the few-layer 2-D thin flakes and the flexible polymer substrates.

Characterization results of the few-layer 2-D thin flakes. Commercial 2-D raw materials with thousands of atom layers were used as starting materials (see FSEM images of raw materials in the Supplementary 


\section{(a) Sandpaper Rubbing Step}

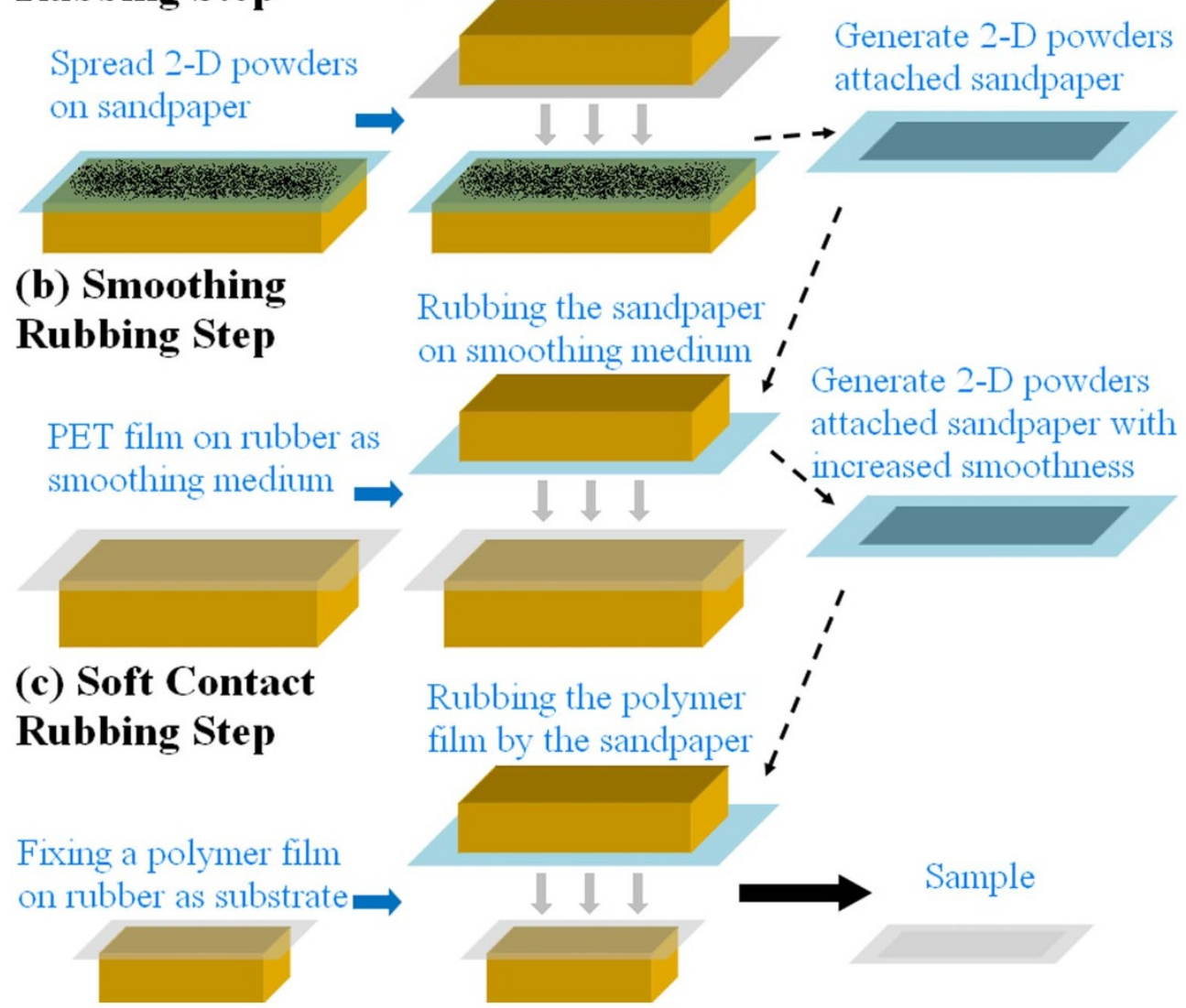

Figure 1 Diagram of the rubbing steps: (a) Step-1, Sandpaper Rubbing Step. (b) Step-2, Smoothing Step. (c) Step-3, Soft Contact Rubbing Step.

Information). Because poly(ethylene terephthalate) (PET) films are very smooth (see the FSEM and AFM images of the raw PET in the Supplementary Information), they were used as flexible polymer substrates for field emission scanning electron microscopy (FSEM), atomic force microscopy (AFM), and other optical characterisations. In addition, polyvinylchloride (PVC) films were used as a flexible polymer substrate for Raman characterisation because the Raman peak positions of the PVC do not interfere with the graphene, $\mathrm{MoS}_{2}$, and $\mathrm{WS}_{2}$ peak positions.

Figure 2a shows that the $514 \mathrm{~nm}$ peak in the Raman spectra changes, with a clear red shift of $2 D$ from approximately $2720 \mathrm{~cm}^{-1}$ for the raw graphite powders to approximately $2690 \mathrm{~cm}^{-1}$ for the few-layer graphene. In addition, the $G / 2 D$ intensity ratio decreased significantly from the raw graphite powders to the few-layer graphene. When comparing these results with previous findings ${ }^{36-38}$, the Raman spectra of graphene suggested that five carbon atom layers occurred in the few layers of graphene on the PVC substrate. Furthermore, the polycrystalline features that were revealed by the $D$ peak originated from the polycrystalline raw graphite powders (see the $D$ peak of the raw graphite powders in Figure 2a and the FSEM image of the raw graphite powders in the Supplementary Information).

Figure $2 \mathrm{~b}$ contains an AFM image of the few-layer graphene on the PET substrate. The thickness of the graphene layer was approximately $1.15 \mathrm{~nm}$. According to previous research ${ }^{37,39}$, graphene monolayers on substrates are approximately $0.65 \sim 0.95 \mathrm{~nm}$ thick. In addition, each additional carbon atom layer is approximately $0.34 \mathrm{~nm}$ thick. Thus, based on the calculated graphene thickness on the PET substrate, 5 or fewer carbon atom layers occurred. The AFM results are consistent with the Raman spectra results.
Figure $2 \mathrm{c}$ shows an AFM image of few-layer h-BN on a PET substrate. The thickness of this layer is approximately $1.70 \mathrm{~nm}$. Based on previous results $s^{5,40}$, a monolayer of $h-B N$ on a substrate is approximately $0.60 \sim 0.90 \mathrm{~nm}$ thick. In addition, each additional hBN atom layer is approximately $0.333 \mathrm{~nm}$ thick. Thus, the calculated thickness of few-layer h-BN on the PET substrate corresponded with to 5 or fewer atom layers.

In addition to the AFM images, Figures $2 \mathrm{~d}$ and e provide separate FSEM images of the few-layer graphene and h-BN on the PET substrate, respectively. The morphology of these films is very similar, which was expected because the graphite and h-BN have similar layered atom structures.

Optical microscopy was used to provide the images of the few-layer graphene and h-BN on the PET substrate (Figures $2 \mathrm{f}$ and g, respectively). These flakes are presented on a scale of tens of micrometres.

Figure 3a shows the change in the trend for the $514 \mathrm{~nm}$ peak in the Raman spectra. Specifically, a $0.4 \mathrm{~cm}^{-1}$ blue shift occurs for the Raman peak position between the raw $\mathrm{MoS}_{2}$ powders and the fewlayer $\mathrm{MoS}_{2}$ on the PVC substrate. In addition, a $0.9 \mathrm{~cm}^{-1}$ red shift of the $A_{1 g}$ Raman peak position between the raw $\mathrm{MoS}_{2}$ powders to the few-layer $\mathrm{MoS}_{2}$ on the PVC substrate occurred. By comparing these results with previously observed Raman spectra of few-layer $\mathrm{MoS}_{2}{ }^{41-43}$, it was observed that no more than $4 \mathrm{MoS}_{2}$ atom layers occurred on the PVC substrate.

An AFM image of the few-layer $\mathrm{MoS}_{2}$ on the PET substrate is presented in Figure 3b. The thickness of this layer is approximately $2.95 \mathrm{~nm}$. According to previous research ${ }^{43,44}$, a monolayer of $\mathrm{MoS}_{2}$ on a substrate is approximately $0.70 \sim 1.00 \mathrm{~nm}$ thick. In addition, each additional $\mathrm{MoS}_{2}$ atom layer is approximately $0.62 \mathrm{~nm}$. Thus, the calculated thickness of the few-layer $\mathrm{MoS}_{2}$ on the PET substrate 
(a)

(b)

(c)

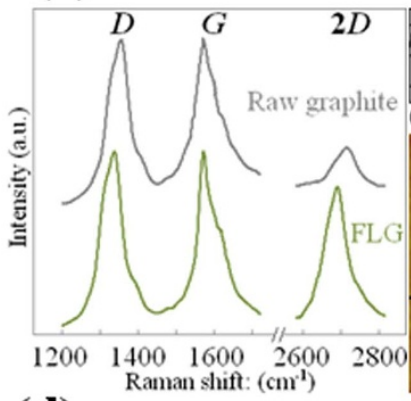

(d)

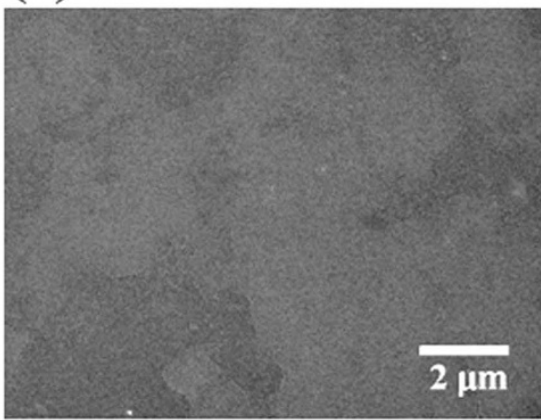

(f)

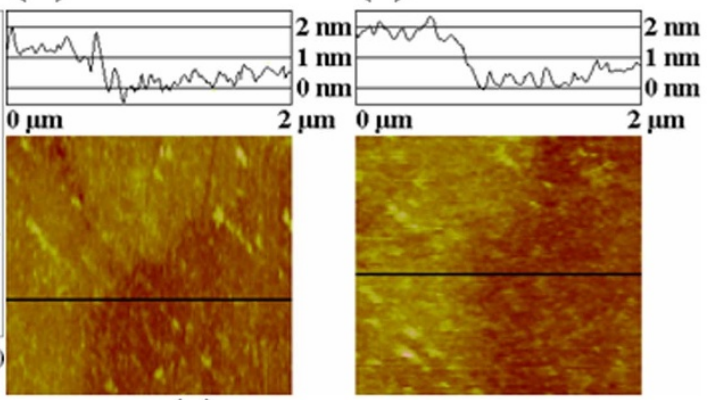

(e)

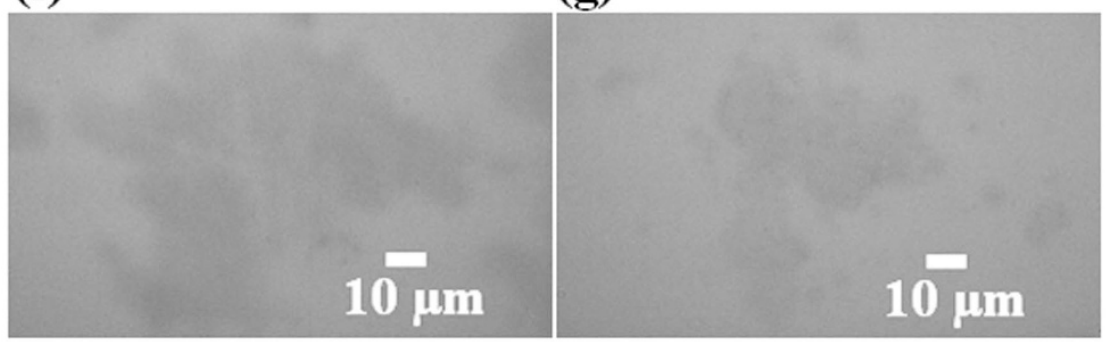

Figure $2 \mid$ (a) Comparison of the Raman spectra. Green and grey lines correspond to the few-layer graphene on PVC substrate and raw graphite powders, respectively. (b) AFM image of few-layer graphene on PET substrate. (c) AFM image of few-layer h-BN on PET substrate. (d) FSEM image of few-layer graphene on PET substrate. (e) FSEM image of few-layer h-BN on PET substrate. (f) Optical image of few-layer graphene on PET substrate. (g) Optical image of few-layer h-BN on PET substrate.

corresponds to 5 or fewer $\mathrm{MoS}_{2}$ atom layers. The AFM results are consistent with the Raman spectra results.

The AFM image of the few-layer $\mathrm{WS}_{2}$ on the PET substrate is shown in Figure 3c. The thickness of this film is approximately $3.05 \mathrm{~nm}$. Based on previous results ${ }^{45}$, a monolayer of $\mathrm{MoS}_{2}$ on a substrate is approximately $1.00 \mathrm{~nm}$ thick. In addition, each additional $\mathrm{WS}_{2}$ atom layer is approximately $0.625 \mathrm{~nm}$. Thus, the calculated thickness of the few-layer $\mathrm{WS}_{2}$ on the PET substrate corresponded with 5 or fewer $\mathrm{WS}_{2}$ atom layers.

The FSEM images of the few-layer $\mathrm{MoS}_{2}$ and $\mathrm{WS}_{2}$ on the PET substrates are presented in Figures $3 \mathrm{~d}$ and e, respectively. The morphologies of these films are very similar, which was expected because $\mathrm{MoS}_{2}$ and $\mathrm{WS}_{2}$ have very similar layered atomic structures.

The optical images of the few-layer $\mathrm{MoS}_{2}$ and $\mathrm{WS}_{2}$ on the PET substrates are provided in Figures $3 \mathrm{f}$ and 6, respectively. These flakes are presented on a scale of tens of micrometres.

Surface stability and related application. One major concern regarding the use of few-layer 2-D thin flakes on flexible polymer substrates is the low surface stability of these products ${ }^{46}$. This low surface stability results from the poor surface adhesion between the polymer films and the few-layer 2-D thin flakes. This poor adhesion generally necessitates an additional protective layer for further applications. In contrast, our previous results have shown that 2-D sheets fabricated on polymer substrates by rubbing have much stronger surface adhesion ${ }^{47-49}$. In the FSEM images (Figures $2 \mathrm{~d}$ and e and Figures $3 \mathrm{~d}$ and e), most of the 2-D thin flakes on the PET substrate have embedded boundaries (boundary lines are ambiguous). This finding agrees with previous findings, including those presented in Ref. 49. In addition, these embedded boundaries offer 2-D thin flakes that are much stronger than other materials that are attached to PET films. In this study, the two types of destructive surface treatments that were used indicated that our samples displayed strong surface stability. In this section, graphene/PET films with similar properties were used in destructive surface treatments (details of the destructive surface treatments are provided in the Methods section). These films were used because their changes in resistivity and transparency changes can be tested accurately. Two pieces of the graphene/PET films were used for the following comparative experiments. (1) The tape test was used to reflect the surface adhesion between the polymer film and functional layer. Next, it was observed that the changes in contrast resistivity and transparency were within $0.1 \%$, which indicated that our samples withstood tape testing. (2) Ultrasonic cleaning is generally an important preparatory part of procedures for several applications. Thus, we studied the effects of ultrasonic cleaning on our samples. After 1 hour of ultrasonic cleaning, the contrast resistivity and transparency changes were within $1.0 \%$. Thus, our samples could withstand a long period of ultrasonic cleaning. 
(a)

(b)

(c)

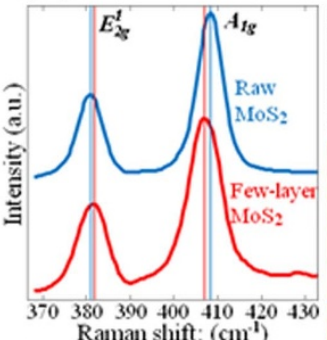

(d)

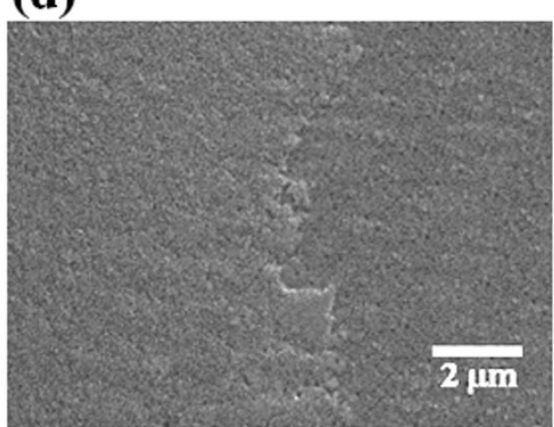

(f)

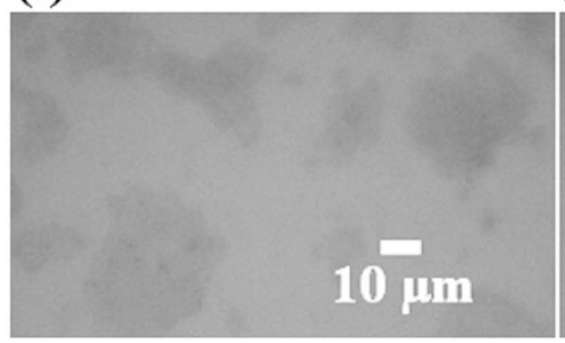

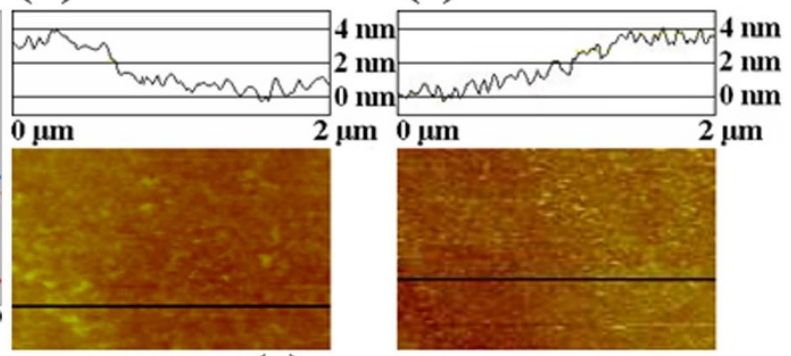

(e)

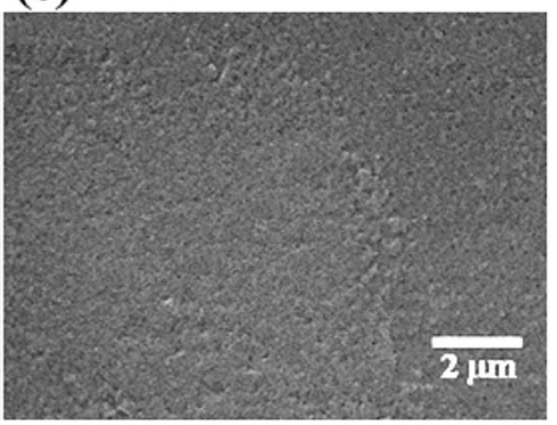

(g)

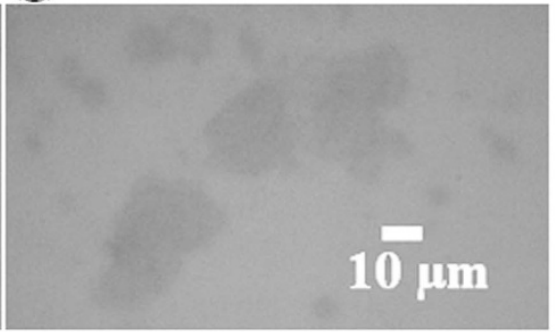

Figure $3 \mid$ (a) Comparison of the Raman spectra. The red and blue lines correspond to the few-layer $\mathrm{MoS}_{2}$ on $\mathrm{PVC}_{\text {substrate and raw } \mathrm{MoS}}$ powders, respectively. (b) AFM image of few-layer $\mathrm{MoS}_{2}$ on PET substrate. (c) AFM image of few-layer $\mathrm{WS}_{2}$ on PET substrate. (d) FSEM image of few-layer MoS 2 on PET substrate. (e) FSEM image of few-layer $\mathrm{WS}_{2}$ on PET substrate. (f) Optical image of few-layer $\mathrm{MoS}_{2}$ on PET substrate. (g) Optical image of few-layer $\mathrm{WS}_{2}$ on PET substrate.

The high surface stabilities of our samples are beneficial for several applications, including flexible transparent conductive films (TCF) with high stability (Figures $4 \mathrm{a}$ and $4 \mathrm{~b}$ ).

Here, the application of our TCFs as transparent strain sensors was investigated. These investigations were made because the strain sensor is an important sensor that is used in many different applications where mechanical deformation is analysed. In addition, graphene networks on polymers can form a type of strain gauge, which combines cheap and scalable production with high sensitivity and novel and attractive properties.

Polyurethane (PU) films were used as substrates for these strain sensors because PU is more sensitive to strain than PET. Figure 4c and $4 \mathrm{~d}$ shows two typical curves of changing resistance $\Delta \mathrm{R}$ as a function of mechanical strain $\varepsilon$. The calculated strain gauge factor (approximately 51) from Figure $4 \mathrm{~d}$ was comparable with the strain gauge factors for the materials that were fabricated from the CVD grown or liquid-exfoliated graphene $e^{50,51}$. In addition, this graphene gauge resulted in a good linear response over a wide range of mechanical strains, which demonstrates the usefulness of the rubbing method for strain sensing.

\section{Discussion}

Friction, which can be imposed on target substrates at room temperature, is closely linked with self-lubricated layered materials, such as graphite. At the end of the 20th century, friction was predicted to be a potential technique for fabricating graphene on target substrates ${ }^{52}$. In 2004, graphene nanosheets with a thickness of between 10 and $100 \mathrm{~nm}$ were fabricated on $\mathrm{SiO}_{2}$ by using a micromechanical manipulation technique ${ }^{53}$, and this technique was closely related to the friction technique. In 2005, two-dimensional atomic crystals were observed on a solid surface after rubbing the layered crystals (including graphite) with solid materials ${ }^{54}$. However, the target substrates that were reported in these studies were solid materials rather than flexible polymers.

In 2012, based on the friction and self-lubrication properties of the two-dimensional layered materials, we proposed rubbing methods for the transfer-free fabrication of two-dimensional nanosheets on flexible polymer substrates at room temperature within minutes of these procedures ${ }^{47,48}$. However, the thicknesses of the nanosheets that were obtained from our previous rubbing methods were approximately 100 to 200 layers (much thicker than mono- and few-layer 2$\mathrm{D}$ thin flakes, which have layers with less than 10 atoms).

In 2013, an innovative smoothing step was developed that uses a self-lubricated smooth rubbing medium that formed by orderly arranged graphite sheets. The function of the smooth rubbing medium surface during the rubbing procedures enabled the fabrication of few-layer graphene (with 2 to 9 carbon atom layers) on the polymer substrate at room temperature ${ }^{49}$. However, this smoothing step can only be conducted for graphene and is not suitable for other fewlayer 2-D thin flakes. 
(a)

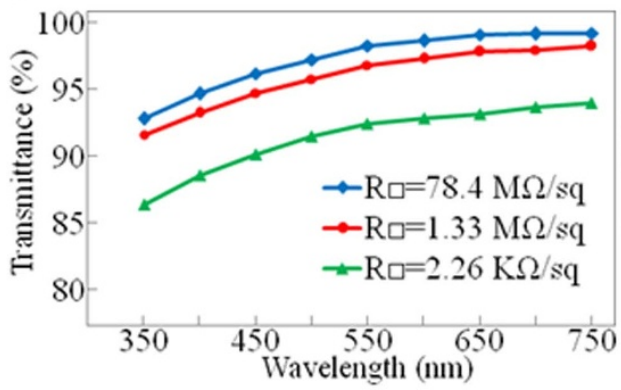

(b)

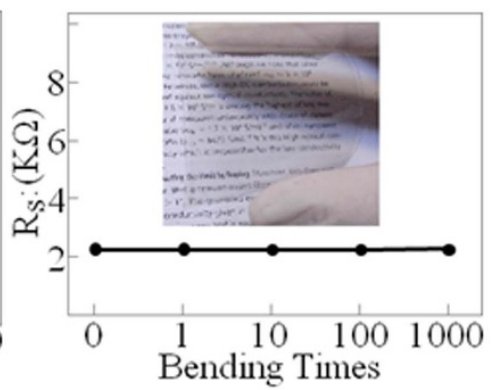

(c)

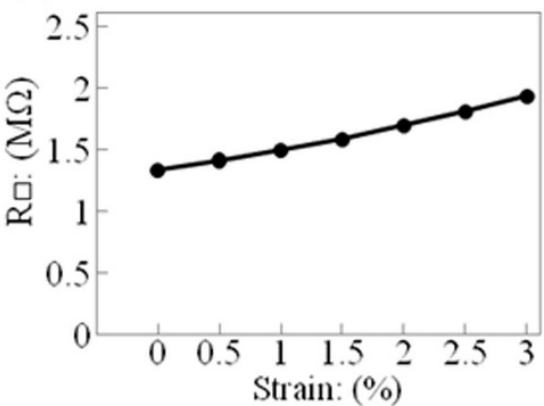

(d)

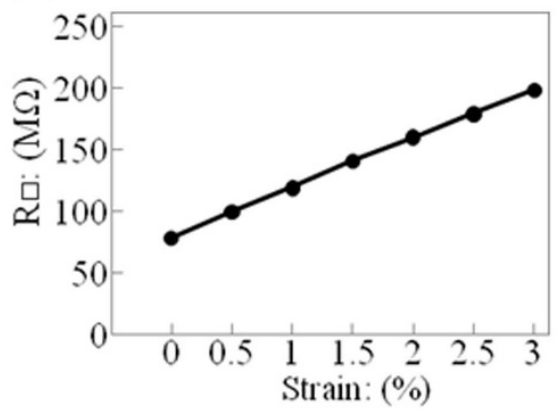

Figure $4 \mid$ (a) Properties of the different flexible TCFs based on graphene/PET (green line) and graphene/PU films (blue and red lines). (b) Photo and stability after bending the graphene/PET on flexible TCF. (c) and (d) depict the changes in resistance as a function of the mechanical strain of graphene/ PU films.

In this study, innovative technical changes have led to the design of a Universal Smoothing Step for different 2-D raw materials (graphite, h-BN, $\mathrm{MoS}_{2}$, and $\mathrm{WS}_{2}$ ) and the formation of self-lubricated smooth rubbing mediums with different kinds of orderly arranged 2-D layered sheets. The Universal Smoothing Step and the resulting self-lubricated smooth rubbing mediums are essential for the fabrication of few-layer 2-D thin flakes on polymer substrates.

A detailed physical relationship between the formation of the smooth rubbing medium and the fabrication of the few-layer 2-D thin flakes was discovered by analysing the FSEM morphology of the sandpaper after Step-1.

In this section, the sandpapers from fabricating all 4 of the fewlayer 2-D thin flakes were tested and compared. These sandpapers were marked as Sandpaper-G, Sandpaper-B, Sandpaper-M and Sandpaper-W, which corresponded with the fabrication of few-layer graphene, few-layer h-BN, few-layer $\mathrm{MoS}_{2}$ and few-layer $\mathrm{WS}_{2}$, respectively.

Figures 5 and 6 clearly illustrate that an important change in the sandpapers' occurred after the "smoothing" procedure in Step-2. In this case, the area of the coarse portion became smaller and the area of the smooth portion became larger.

In the smooth areas, the 2-D sheets covered the sandpaper and served as a smooth rubbing medium (Figures $5 \mathrm{e}$ and $\mathrm{f}$ and $6 \mathrm{e}$ and $\mathrm{f}$ ). However, in the coarser portions, the attachment of the 2-D sheets to the sandpaper was disorderly.

The smooth rubbing mediums and the 2-D sheets of sandpaper were worked together during the rubbing process in Step-3. The 2-D sheets were rubbed onto the polymer substrates by using "soft pressure" during Step-3. These 2-D sheets were rubbed with the smooth rubbing mediums during Step-3. The self-lubricating property of the smooth rubbing medium is important for fabricating few-layer 2-D thin flakes on polymer films. Because if the rubbing mediums have a high friction coefficient, the 2-D sheets on the polymer substrates can be rubbed away by them.

Similar surface fractal evolutions were observed in our previous studies for all of the rubbing surfaces that were used in this study (a)

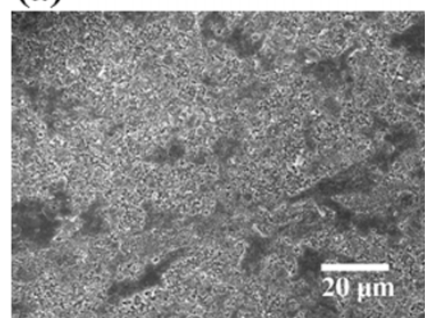

(b) (c)

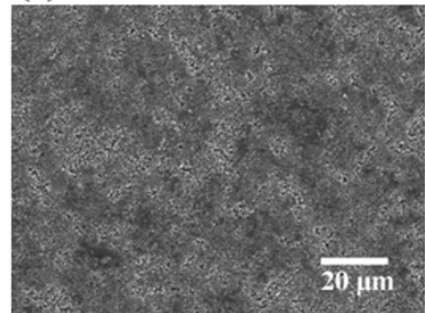

(e)

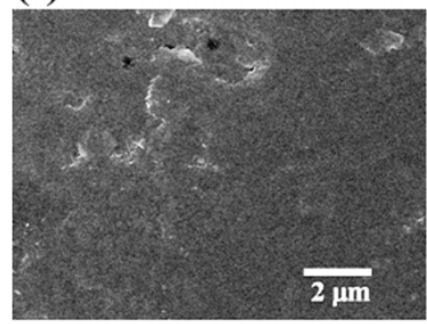

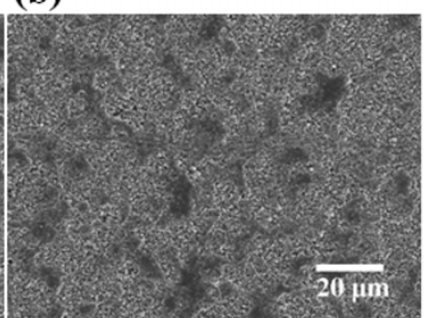

(d)

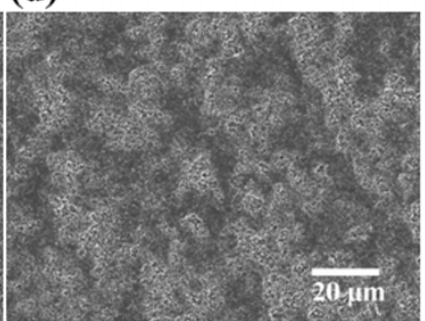

(f)

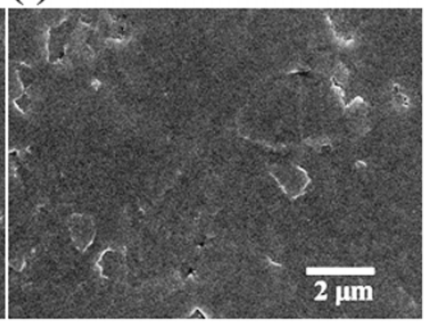

Figure $5 \mid$ (a) FSEM image of Sandpaper-G before Step-2. (b) FSEM image of Sandpaper-B before Step-2. (c) FSEM image of Sandpaper-G after Step-2. (d) FSEM image of Sandpaper-B after Step-2. (e) High-magnification FSEM image of the smooth rubbing medium of Sandpaper-G after Step-2.

(f) High-magnification FSEM image of the smooth rubbing medium of Sandpaper-B after Step-2. 
(a)

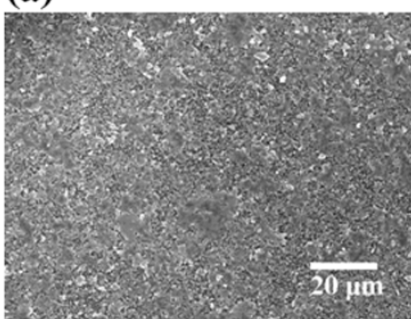

(c)

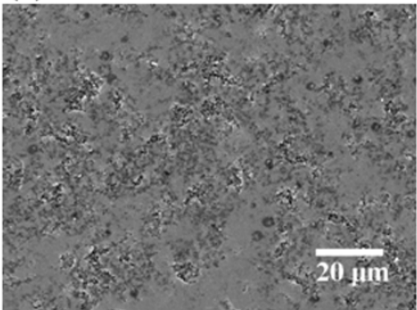

(e)

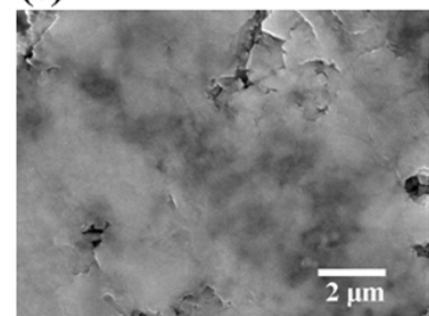

(f)

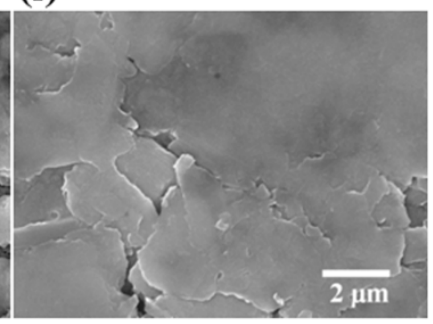

Figure 6 | (a) FSEM image of Sandpaper-M before Step-2. (b) FSEM image of Sandpaper-W before Step-2. (c) FSEM image of Sandpaper-M after Step-2. (d) FSEM image of Sandpaper-W after Step-2. (e) Highmagnification FSEM image of the smooth rubbing medium of Sandpaper$M$ after Step-2. (f) High-magnification FSEM image of the smooth rubbing medium of Sandpaper-W after Step-2.

(Table 1). These evolutions potentially cause changes in the ratio of coarse portions to smooth portions. The ratio of the coarse portions to smooth portions is important in Step-3. If there were no "smoothing" procedures, the coarse portions would be very large relative to the smooth portions (Figures $5 \mathrm{a}$ and $\mathrm{c}$ and $6 \mathrm{a}$ and $\mathrm{c}$ ). In addition, too many 2-D sheets would be rubbed on the polymer substrates. Finally, the relatively small smooth rubbing mediums would not be sufficient for rubbing the excess 2-D sheets to achieve a desired thickness of only a few layers of 2-D thin flakes. Thus, without these smoothing procedures, more than 10 layers of 2-D nanosheets would occur on the polymer substrates. In contrast, if the "smoothing" time in Step-3 is very long, the areas of the coarse portions are small relative to the areas of the smooth rubbing mediums (Figures $5 \mathrm{~b}$ and $\mathrm{d}$ and $6 \mathrm{~b}$ and d). Thus, the amount of the 2-D sheet that was rubbed on the polymer substrate was small. In addition, the relatively large smooth rubbing mediums can be used to reduce the 2-D sheets to 2-D thin flakes. In addition, the fractal dimensions of the few-layer 2-D thin flakes were similar to the fractal dimensions of the smooth rubbing surfaces (Table 2), which indicated that a linkage occurred between fractal evolution and growth in the few-layer 2-D thin flakes.

Additional comparative results on the specific conditions of soft contact rubbing step are discussed in the Supplementary Information.

Table 2 | Calculated fractal dimensions of the few-layer 2-D thin flakes on PET

\begin{tabular}{|lcccc|} 
& Graphene & h-BN & $\mathrm{MoS}_{2}$ & $\mathrm{WS}_{2}$ \\
\hline $\begin{array}{l}\text { Fractal dimensions of 2-D thin } \\
\text { flakes on PET }\end{array}$ & 1.287 & 1.317 & 1.305 & 1.292 \\
\hline
\end{tabular}

The high resolution FSEM images (Figure 5e, f and Figure 6e, f) clearly show the presence of defects, which may cause existence of small flakes and affect the film surface quality of samples. Since defects can have a strong influence on the properties of 2-D materials based films ${ }^{55,56}$, and on the other side, the control of defects allows making preparation of 2-D materials based films with novel properties $^{56,57}$. Several potential methods as following may bring further improvements on the quality and deserve future studies: (1) using raw 2-D materials with higher quality, or pre-treat (e.g. ultrasonic cleaning and centrifugation) the raw materials to obtain more homogeneous starting materials, (2) Post-processing on the sandpaper surfaces after smooth rubbing step to obtain smooth rubbing mediums with higher quality.

According to the experimental and theoretical reports on the friction properties of mono-and few-layer 2-D materials, double to several atom layers of 2-D materials can lead to a significantly better lubrication property than single atom layer $2-\mathrm{D}$ materials ${ }^{58,59}$. These could result in the friction increment upon thinning of the 2-D thin flakes when in the range of few-layer. And the development progress of rubbing method from 2012 did show that it becomes more and more difficult to remove layers when approaching mono-layer 2-D thin flake. Thus, the limit on the atom layer numbers of 2-D thin flakes fabricated from current rubbing procedures and the special points of further improvements to get mono-layer 2-D flakes are needed to be explored.

In summary, we fabricated different types of few-layer 2-D thin flakes directly on flexible polymer substrates by using commercial 2$\mathrm{D}$ raw materials with thousands of atom layers. During this fabrication process, rubbing procedures were performed for several minutes at room temperature without any transfer step. This rubbing method has the following important advantages: (1) producing a variety of few-layer 2-D thin flakes, (2) the rapid fabrication procedures (minutes) at room temperature, (3) the direct fabrication on flexible polymer substrates without any transfer step, (4) low raw material costs, and (5) a strong surface adhesion between the few-layer 2-D thin flakes and the flexible polymer substrates. The sizes of the few-layer 2-D thin flakes were approximately tens of micrometres. The physical relationship between the formation of the smooth rubbing mediums and the few-layer 2-D thin flakes is discussed based on the morphological changes of the sandpaper surfaces after each step. This study provides highly efficient, low cost, and direct fabrication of few-layer 2-D thin flakes at room temperature. The resulting few-layer 2-D thin flakes strongly adhered to the flexible polymer substrates. These results demonstrated that rubbing methods are potentially important in the fabrication and application of nanomaterials.

\section{Methods}

Raw materials. Polytetrafluoroethylene (PTFE) films are needed to assist the Sandpaper Rubbing Step (Step-1), and PET films are needed as smoothing mediums. PET, PVC and PU films are used as flexible polymer substrates. Several pieces of

Table 1 | Calculated fractal dimensions of the sandpaper surfaces before and after Step-2

\begin{tabular}{lccc} 
& Graphene & h-BN & MoS $_{2}$ \\
\hline Fractal dimensions of smooth parts on the sandpaper surface before Step-2 & 1.302 & 1.280 & 1.343 \\
Fractal dimensions of coarse parts on the sandpaper surface after Step-2 & 1.293 & 1.307 \\
\hline
\end{tabular}


polishing sandpapers are required for the method. A series of commercial graphite, h$\mathrm{BN}, \mathrm{MoS}_{2}, \mathrm{WS}_{2}$ powders are needed as raw materials.

Step-1, Sandpaper Rubbing Step. The commercial raw powders were rubbed between PTFE films and polishing sandpaper (Figure 1a) by applying gentle pressure $(0.1 \mathrm{MPa})$ for 20 seconds. This gentle pressure was transferred by commercial rubber (shown as the yellow rectangles in Figure 1a) that was fixed on commercial glass blocks. In addition, this step was used to apply a thin and uniform layer of powder on the polishing sandpapers. The rubbing track can be described as "revolution without self-rotation" (like the rubbing track from our previous studies ${ }^{47-49}$ ). The rubbing rate was $200 \mathrm{rpm}$ and the rubbing diameter was $0.5 \mathrm{~cm}$.

Step-2, Smoothing Step. After fixing a PET film on commercial rubber (the light grey piece on the yellow rubber in Figure $1 b$ ), we rubbed the polishing sandpaper on the PET film by applying soft pressure $(0.04 \mathrm{MPa})$ over different periods $(50-300$ seconds for the different 2-D raw materials). This soft pressure was transferred by commercial rubbers (shown as the yellow rectangles in Figure 1b) that were fixed on commercial glass blocks. The rubbing track that was used in this study corresponds with the rubbing track that was used in our previous studies ${ }^{47-49}$. Here, a rubbing rate of $200 \mathrm{rpm}$ and a rubbing diameter of $0.5 \mathrm{~cm}$ were used. Following this rubbing process, the polishing sandpaper surface had different degrees of smoothness that corresponded with the rubbing periods.

Step-3, Soft Contact Rubbing Step. After fixing a polymer film (PET or PVC or PU) on a commercial piece of rubber (the light grey piece on yellow rubber in Figure 1c), the polymer film was rubbed with the polishing sandpaper by applying soft pressure $(0.1 \mathrm{MPa})$ for 40 seconds. This soft pressure was transferred by commercial rubbers (shown as the yellow rectangles in Figure 1c) that were fixed on commercial glass blocks. The rubbing track that was used in this study was the same that was used in our previous studies ${ }^{47-49}$. A rubbing rate of $200 \mathrm{rpm}$ and a rubbing diameter of $0.5 \mathrm{~cm}$ were used. This rubbing process was used to fabricate the sample.

After Step-3, the samples were washed in deionised water in an ultrasonic bath for $1 \mathrm{~min}$

Surface Destructive Treatments. (1) Bending test: The sample was bent in a semicircular shape for 2 seconds. This process was repeated between 1 and 1000 times. (2) Tape test: A commercial tape was pasted and pressed on one piece of the sample at a pressure of $1 \mathrm{MPa}$ for $30 \mathrm{~min}$ before tearing the tape off. (3) Long term ultrasonic cleaning test: One piece of the sample was placed an ultrasonic bath containing deionised water and cleaned for 1 hour.

Materials characterizations. Raman spectra of few-layer graphene and few-layer $\mathrm{MoS}_{2}$ on PVC films were characterized by Raman spectrometry $(514 \mathrm{~nm})$. The thicknesses of few-layer 2-D thin flakes on PET films were tested by Atomic Force Microscopy (AFM). The sizes and distribution of few-layer graphene on PET films were observed by Optical microscopy. The morphology of few-layer 2-D thin flakes on PET films and the morphology changes of polishing sandpaper surface were characterized by Field emission Scanning Electron Microscopy (FSEM). Sheet resistance and optical transparency measurements of flexible transparent conductive films (few-layer graphene/PET and few-layer graphene/PU films) were taken by Four-probe tester and UV-Vis Spectrophotometry, respectively.

Strain sensors fabrication and characterizations. Such sensors were fabricated by printing silver pads $(4 \mathrm{~cm} \times 1.0 \mathrm{~cm})$ onto $4 \mathrm{~cm} \times 6.0 \mathrm{~cm}$ sized few-layer graphene/ PU films' surfaces to define a patterned specific shape. The strain gauge factors were calculated by testing the resistivity changes under different strain ratios as Ref. 50 and Ref. 51.

Fractal dimension calculations. The surface evolution behavior on sandpaper surfaces during Step-3 were studied using the fractal power law equations ${ }^{60}$.

$$
\begin{gathered}
N(\varepsilon)=k \varepsilon^{-D}, \\
N(n \varepsilon)=n^{-D} N(\varepsilon),
\end{gathered}
$$

Where $\varepsilon$ is the side length unit of box for coverage of boundary, $N(\varepsilon)$ and $N(n \varepsilon)$ are the numbers of boxes (with different side lengths: $n \varepsilon$ ) needed to cover the boundary, $D$ is the fractal dimension. In this study, $n=1,2,3,4,5$ were used to calculate value of $D$ by box-counting method ${ }^{60}$.

1. Neto, A. H. C. \& Novoselov, K. S. New directions in science and technology: twodimensional crystals. Rep. Prog. Phys. 74, 082501 (2011).

2. Bonaccorso, F., Lombardo, A., Hasan, T., Sun, Z., Colombo, L. \& C. Ferrari, A. C Production and processing of graphene and $2 \mathrm{~d}$ crystals. Mater. Today 15, 564-589 (2012).

3. Novoselov, K. S., Fal'ko, V. I., Colombo, L., Gellert, P. R., Schwab, M. G. \& Kim, K. A roadmap for graphene. Nature 490, 192-200 (2012).

4. Liang, Y. T. \& Hersam, M. C. Towards Rationally Designed Graphene-Based Materials and Devices. Macromol. Chem. Phys. 213, 1091-1100 (2012).

5. Pakdel, A., Zhi, C., Bando, Y. \& Golberg, D. Low-dimensional boron nitride nanomaterials. Mater. Today 15, 256-265 (2012).
6. Xu, M., Liang, T., Shi, M. \& Chen, H. Graphene-Like Two-Dimensional Materials. Chem. Rev. 113, 3766-3798 (2013).

7. Rao, C. N. R. \& Nag, A. Inorganic Analogues of Graphene. Eur. J. Inorg. Chem. 2010, 4244-4250 (2010).

8. Butler, S. Z. et al. Progress, Challenges, and Opportunities in Two-Dimensional Materials Beyond Graphene. ACS Nano 7, 2898-2926 (2013).

9. Huang, X., Zeng, Z. \& Zhang, H. Metal dichalcogenide nanosheets: preparation, properties and applications. Chem. Soc. Rev. 42, 1934-1946 (2013).

10. Wang, Q. H., K.-Zadeh, K., Kis, A., Coleman, J. N. \& Strano, M. S. Electronics and optoelectronics of two-dimensional transition metal dichalcogenides. Nat. Nanotechnol. 7, 699-712 (2012).

11. Matte, H. S. S. R. et al. $\mathrm{MoS}_{2}$ and $\mathrm{WS}_{2}$ Analogues of Graphene. Angew. Chem. Int. Ed. 49, 4059-4062 (2010).

12. Koski, K. J., \& Cui, Y. The New Skinny in Two-Dimensional Nanomaterials. ACS Nano. 7, 3739-3743 (2013).

13. Balandin, A. A. Thermal properties of graphene and nanostructured carbon materials. Nat. Mater. 10, 569-581 (2011)

14. Sreeprasad, S. T. \& Berry, V. How Do the Electrical Properties of Graphene Change with its Functionalization? Small 9, 341-350 (2013).

15. Santos, E. J. G. \& Kaxiras, E. Electric-Field Dependence of the Effective Dielectric Constant in Graphene. Nano Lett. 13, 898-902 (2013).

16. Baskin, A. \& Král, P. Electronic structures of porous Nanocarbons. Sci. Rep. 1, 36 (2011).

17. Li, H. \& Zeng, X. Wetting and Interfacial Properties of Water Nanodroplets in Contact with Graphene and Monolayer Boron_Nitride Sheets. ACS Nano 6, 2401-2409 (2012).

18. Chhowalla, M., Shin, H. S., Eda, G., Li, L., Loh, K. P. \& Zhang, H. The chemistry of two-dimensional layered transition metal dichalcogenide nanosheets. Nat. Chem. 5, 263-275 (2013).

19. Shi, H., Zhang, Y. \& Yakobson, B. I. Quasiparticle band structures and optical properties of strained monolayer $\mathrm{MoS}_{2}$ and $\mathrm{WS}_{2}$. Phys. Rev. B 87, 155304 (2013).

20. Yang, S., Sun, Y., Chen, L., Hernandez, Y., Feng, X. \& Müllen, K. Porous Iron Oxide Ribbons Grown on Graphene for High-Performance Lithium Storage. Sci. Rep. 2, 427 (2012).

21. Bao, Q. \& Loh, K. P. Graphene Photonics, Plasmonics, and Broadband Optoelectronic Devices. ACS Nano 6, 3677-3694 (2012).

22. Pu, J., Yomogida, Y., Liu, K., Li, L., Iwasa, Y. \& Takenobu, T. Highly Flexible $\mathrm{MoS}_{2}$ Thin-Film Transistors with Ion Gel Dielectrics. Nano Lett. 12, 4013-4017 (2012).

23. Choi, M. S. et al. Controlled charge trapping by molybdenum disulphide and graphene in ultrathin heterostructured memory devices. Nat. Commun. 4, 1624 (2013).

24. Rogers, J. A., Someya, T. \& Huang, Y. Materials and Mechanics for Stretchable Electronics. Science 327, 1603-1607 (2010).

25. Edwards, R. S. \& Coleman, K. S. Graphene synthesis: relationship to applications. Nanoscale 5, 38-51 (2013).

26. Kang, J., Shin, D., Bae, S. \& Hong, B. H. Graphene transfer: key for applications. Nanoscale 4, 5527-5537 (2012).

27. Nicolosi, V., Chhowalla, M., Kanatzidis, M. G., Strano, M. S. \& Coleman, J. N. Liquid Exfoliation of Layered Materials. Science 340, 6139-6156 (2013)

28. Coleman, J. N. et al. Two-Dimensional Nanosheets Produced by Liquid Exfoliation of Layered Materials. Science 331, 568-571 (2011).

29. Park, S. \& Ruoff, R. S. Chemical methods for the production of graphenes. Nat. Nanotechnol. 4, 217-224 (2009)

30. Mattevi, C., Kim, H. \& Chhowalla, M. A review of chemical vapour deposition of graphene on copper. J. Mater. Chem. 21, 3324-3334 (2011).

31. Wei, D., Wu, B., Guo, Y., Yu, G. \& Liu, Y. Controllable Chemical Vapor Deposition Growth of Few Layer Graphene for Electronic Devices. Acc. Chem. Res. 46, 106-115 (2013).

32. Zhan, Y., Liu, Z., Najmaei, S., Ajayan, P. M. \& Lou, J. Large-Area Vapor-Phase Growth and Characterization of $\mathrm{MoS}_{2}$ Atomic Layers on $\mathrm{SiO}_{2}$ Substrate. Small 8 966-971 (2012)

33. Ma, L., Ren, W., Dong, Z., Liu, L. \& Cheng, H. Progress of graphene growth on copper by chemical vapor deposition: Growth behavior and controlled synthesis. Chin. Sci. Bull. 57, 2995-2999 (2012).

34. Mao, S., Yu, K., Chang, J., Steeber, D. A., Ocola, L. E. \& Chen, J. Direct Growth of Vertically-oriented Graphene for Field-Effect Transistor Biosensor. Sci. Rep. 3, 1696 (2013).

35. Kwak, J. et al. Near room-temperature synthesis of transfer-free graphene films. Nat. Commun. 3, 645 (2012).

36. Ferrari, A. C. et al. Raman Spectrum of Graphene and Graphene Layers. Phys. Rev. Lett. 97, 187401 (2006)

37. Hao, Y. et al. Probing Layer Number and Stacking Order of Few-Layer Graphene by Raman Spectroscopy. Small 6, 195-200 (2010).

38. Rafiee, J. et al. Wetting transparency of graphene. Nat. Mater. 11, 217-222 (2012).

39. Novoselov, K. S. et al. Electric Field Effect in Atomically Thin Carbon Films. Science 306, 666-669 (2004).

40. Shi, Y. et al. Synthesis of Few-Layer Hexagonal Boron Nitride Thin Film by Chemical Vapor Deposition. Nano Lett. 10, 4134-4139 (2010).

41. Li, H. et al. From Bulk to Monolayer $\mathrm{MoS}_{2}$ : Evolution of Raman Scattering. $A d v$. Funct. Mater. 22, 1385-1390 (2012). 
42. Najmaei, S., Liu, Z., Ajayan, P. M. \& Lou, J. Thermal effects on the characteristic Raman spectrum of molybdenum disulfide $\left(\mathrm{MoS}_{2}\right)$ of varying thicknesses. Appl. Phys. Lett. 100, 013106 (2012).

43. Lee, C., Yan, H., Brus, L. E., Heinz, T. F., Hones, J. \& Ryu, S. Anomalous Lattice Vibrations of Single and Few-Layer $\mathrm{MoS}_{2}$. ACS Nano 4, 2695-2700 (2010).

44. Lopez-Sanchez, O., Lembke, D., Kayci, M., Radenovic, A. \& Kis, A. Ultrasensitive photodetectors based on monolayer $\mathrm{MoS}_{2}$. Nat. Nanotechnol. 8, 497-501 (2013).

45. Berkdemir, A. et al. Identification of individual and few layers of $\mathrm{WS}_{2}$ using Raman Spectroscopy. Sci. Rep. 3, 1755 (2013).

46. Wassei, J. K. \& Kaner, R. B. Graphene, a promising transparent conductor. Mater. Today 13, 52-59 (2010).

47. Yu, Y. et al. Ultrafast room temperature wiping-rubbing fabrication of graphene nanosheets as flexible transparent conductive films with high surface stability. Appl. Phys. Lett. 101, 023119 (2012).

48. Yu, Y. et al. Universal ultrafast sandpaper assisting rubbing method for room temperature fabrication of two-dimensional nanosheets directly on flexible polymer substrate. Appl. Phys. Lett. 101, 073113 (2012).

49. Yu, Y. et al. Surface fractal evolution induced rubbing for rapid room temperature and transfer-free fabrication of graphene on flexible polymer substrate. Appl. Phys. Lett. 103, 011601 (2013).

50. Hempel, M., Nezich, D., Kong, J. \& Hofmann, M. A Novel Class of Strain Gauges Based on Layered Percolative Films of 2D Materials. Nano Lett. 12, 5714-5718 (2012).

51. Bae, S., Lee, Y., Sharma, B. K., Lee, H., Kim, J. \& Ahn, J. Graphene-based transparent strain sensor. Carbon 51, 236-242 (2013).

52. Lu, X., Yu, M., Huang, H. \& Ruoff, R. S. Tailoring graphite with the goal of achieving single sheets. Nanotechnol. 10, 269-272 (1999).

53. Zhang, Y., Small, J. P., Pontius, W. V. \& Kim, P. Fabrication and electric-fielddependent transport meaturements of mesoscopic graphite devices. Appl. Phys. Lett. 86, 073104 (2005).

54. Novoselov, K. S. et al. Two-dimensional atomic crystals. Proc. Natl. Acad. Sci. 102 10451-10453 (2005).

55. Zou, X., Liu, Y. \& Yakobson, B. I. Predicting Dislocations and Grain Boundaries in Two-Dimensional Metal-Disulfides from the First Principles. Nano Lett. 13, 253-258 (2013).

56. Eckmann, A. et al. Probing the Nature of Defects in Graphene by Raman Spectroscopy. Nano Lett. 12, 2925-2930 (2012).

57. Lahiri, J., Lin, Y., Bozkurt, P., Oleynik, I. I. \& Batzill, M. An extended defect in graphene as a metallic wire. Nat. Nanotechnol. 5, 326-329 (2010).
58. Lee, C. et al. Frictional Characteristics of Atomically Thin Sheets. Science 328, 76-80 (2010)

59. Filleter, T. et al. Friction and Dissipation in Epitaxial Graphene Films. Phys. Rev. Lett. 102, 086102 (2009).

60. Ling, F. The Possible Role of Fractal Geometry in Tribology. Tribol. Trans. 32, 497-505 (1989)

\section{Acknowledgments}

This work was supported by Award of Academic Newcomers for Doctoral Students (Ministry of Education of the People's Republic of China), Key Project of Outstanding Doctoral Dissertation Foundation of Huazhong University of Science and Technology, National Natural Science Foundation of China (No. 61274115), and the National Science and Technology Support Program (2012BA113B00). We acknowledge Analytical and Testing Center of Huazhong University of Science and Technology for testing our samples.

\section{Author contributions}

Y.Y. and S.J. conducted the research. Y.Y. fabricated the 2-D thin flakes on polymers. Y.Y., W.Z. and X.M. carried out the materials characterizations. Y.Y., Y.Z., and G.Z. made the strain sensors and did the device testing. Y.Y. and S.L. did the calculations in this research. Y.Y. drafted the paper and the Supplementary Information. All authors contributed to the final paper.

\section{Additional information}

Supplementary information accompanies this paper at http://www.nature.com/ scientificreports

Competing financial interests: The authors declare no competing financial interests. How to cite this article: Yu, Y. et al. Room temperature rubbing for few-layer two-dimensional thin flakes directly on flexible polymer substrates. Sci. Rep. 3, 2697; DOI:10.1038/srep02697 (2013).

(c) (i) $(-)$ This work is licensed under a Creative Commons AttributionBY NC ND NonCommercial-NoDerivs 3.0 Unported license. To view a copy of this license, visit http://creativecommons.org/licenses/by-nc-nd/3.0 
SUBJECT AREAS: $\quad$ ERRATUM: Room temperature rubbing for few-layer two-dimensional thin flakes SURFACES, INTERFACES

AND THIN FILMS

GRAPHENE directly on flexible polymer substrates

Yan Yu, Shenglin Jiang, Wenli Zhou, Xiangshui Miao, Yike Zeng, Guangzu Zhang \& Sisi Liu

TWO-DIMENSIONAL MATERIALS

DESIGN, SYNTHESIS AND PROCESSING

A file entitled 'Supplementary Dataset 1' was inadvertently published as Supplementary Information with this Article. This file has now been removed.

SCIENTIFIC REPORTS:

$3: 2697$

DOI: $10.1038 /$ srep02697

(2013)

Published:

18 September 2013

Updated:

21 November 2013 